\title{
An unusual cause for diplopia: acquired Brown's syndrome
}

\author{
G N Fuller, T D Matthews, R N Maini, C Kennard
}

Brown's syndrome, the limitation of upgaze in an adducted eye relating to a malfunction of the superior oblique tendon and trochlear complex, is well recognised in the ophthalmological literature ${ }^{1}$ but is rarely reported in the neurological literature ${ }^{2}$ and does not appear in a range of standard neurological textbooks.

We report a young woman with Brown's syndrome who was initially thought to have a partial third nerve palsy. The correct identification of this syndrome prevented inappropriate invasive neuroradiological investigations being carried out. Subsequent investigations found her to have rheumatoid factor positive palindromic rheumatism.

A previously fit 34 year old woman presented with a history of worsening painful diplopia on right gaze. Eight days previously she had first noted a continuous pain in the inner, upper aspect of her left orbit. Two days later she became aware of diplopia on looking to the right, especially on looking up, where the images were vertically displaced. Looking up and to the right increased the pain. Over the next four days the diplopia worsened, occurring on smaller movements of her eyes to the right.

General examination at presentation was normal. Corrected visual acuity was $6 / 6$ on the right and $6 / 9$ on the left. Pupils were normal. There was a slight exophoria in the primary position. Diplopia was present on upgaze, most pronounced in right gaze, with minimal diplopia on right downgaze. Elevation of the left eye was correspondingly limited in adduction, with minimal limitation of depression in adduction. There was no limitation of elevation in abduction. There was downshoot of the left eye on adduction with slight restriction due to pain. Movements of the right eye were normal with the exception of compensatory overaction of elevation in abduction. Fundoscopy was unremarkable. Neurological examination was normal.

The painful ophthalmoplegia was initially interpreted as being due to a progressive partial third nerve palsy particularly involving the inferior oblique muscle. Computed tomography and orbits and a tensilon test were performed. Both of these were normal.

A Hess chart was obtained (figure), which identifies the limitation of elevation in adduction of the left eye with associated compensatory overaction of the right superior rectus. There was minimal limitation of left superior oblique action and this is consistent with the minimal diplopia reported in downgaze. This appearance of the Hess chart taken with the clinical picture is diagnostic of restriction of the superior oblique muscle (Brown's syndrome).

Full blood count, erythrocyte sedimentation rate, and $C$ reactive protein and glucose concentrations were normal. Antinuclear antigen was borderline positive $(+)$, with a diffuse pattern. Tests for antibodies to double stranded DNA and other diagnostically specific nuclear and cytoplasmic antigens were all negative. Rheumatoid factor was positive at 1/15 120. Formal rheumatological assessment found a 13 year history of intermittent painful ankle swelling, occurring for two to three days at a time, and a two year history of right shoulder pain. Examination showed a painful arc syndrome affecting the right shoulder. These findings led to a diagnosis of "palindromic rheumatism", which in a proportion of patients, may evolve into a fully expressed disorder such as rheumatoid arthritis.

She declined a local injection of steroids to the trochlea, and was therefore managed conservatively. On review two weeks later her diplopia was clearly improving and at 10 weeks (figure) she had minimal diplopia. At four months full recovery had occurred.

This patient had diplopia due to restriction of superior oblique action, with pain at the site of the trochlea, no structural orbital lesion, and a serological marker for rheumatoid arthritis. This constellation of findings is diagnostic of inflammatory Brown's syndrome.

The initial assessment misinterpreted the limitation of elevation in adduction (the primary direction of action of the inferior oblique muscle), and a reluctance to adduct the eye as indicating a partial third nerve lesion. As the history was progressive over days, investigations for a compressive third nerve lesion were initiated and angiography was considered. The recognition of this as acquired Brown's syndrome prevented inappropriate invasive investigation. Patients with Brown's syndrome may also present with intermittent painless diplopia. It should therefore be considered in the differential diagnosis of ocular myasthenia gravis. In this situation a Hess chart, which is characteristic in Brown's syndrome, ${ }^{3}$ should be performed.

Harold Whaley Brown first described "the 
Top. Hess chart at presentation. This shows the limitation of movement in the direction of action of the inferior oblique muscle in the left eye and compensatory overaction of the superior rectus muscle in the right eye. There is also some limitation of action of superior oblique muscle in the left eye. Bottom. Repeat Hess chart 10 weeks later showing pronounced improvement.
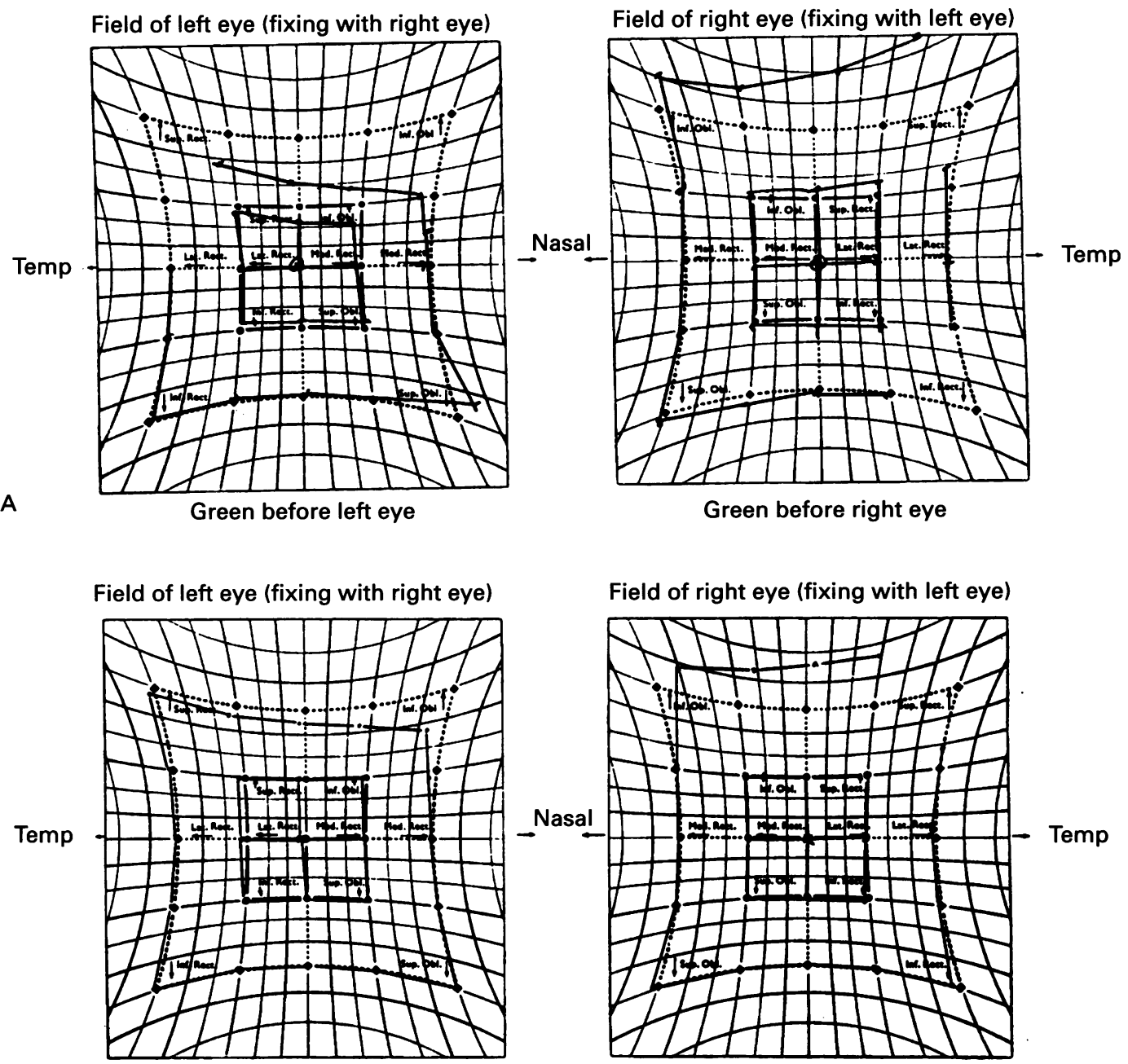

Green before left eye

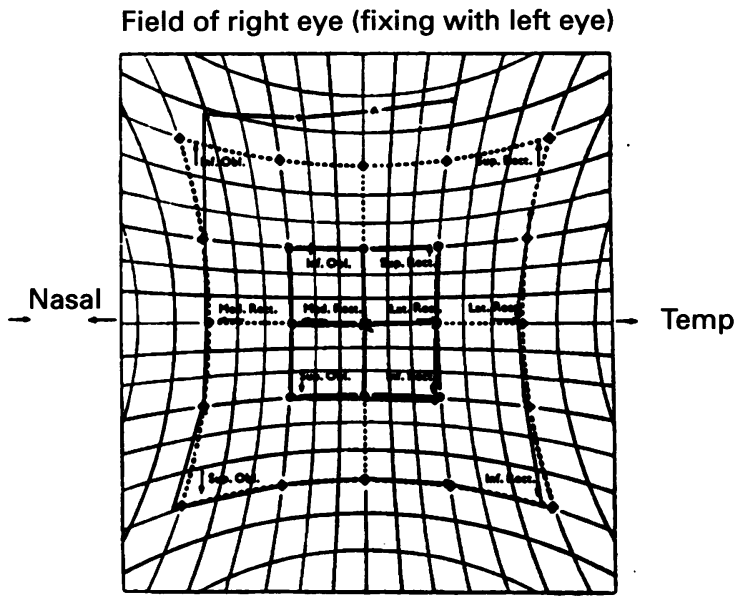

Green before right eye superior oblique tendon sheath syndrome" in 1949 , characterised by restriction of active and passive elevation in adduction. ${ }^{4}$ Most of the early literature was concerned with the congenital form of this syndrome with some controversy as to the underlying mechanism. ${ }^{1}$

It has been reported in rheumatological disorders: rheumatoid arthritis, ${ }^{5}$ juvenile chronic arthritis (Still's Disease), ${ }^{3}$ and in adults with previous Still's disease. ${ }^{5}$ In these circumstances a local tenosynovitis is thought to be the mechanism. In all previously reported cases there was preceding or concurrent evidence of systemic or articular manifestations of rheumatological disease. Our case represents the first report of a patient with acquired Brown's syndrome as the presenting manifestation of rheumatological disease.

In most patients with Brown's syndrome due to a rheumatological cause there is a full recovery in weeks to months. Treatment with either systemic or local steroids and nonsteroidal anti-inflammatory drugs is usually given. Our patient shows that recovery can occur without treatment.

This syndrome has also been reported in association with local pathology in the orbit near the trochlea or superior oblique tendon. It is therefore advisable to image the orbits in suspected cases.

In view of the diverse aetiologies and multiple proposed pathogenetic mechanisms it may be more appropriate to consider limitation of elevation in adduction as Brown's sign rather than Brown's syndrome.

When presented with a patient with apparent weakness of the inferior oblique muscle it is essential to consider a functional restriction of the superior oblique muscle as the underlying cause. This will not only prevent inappropriate neuroradiological investigation but may lead to the correct identification of the underlying pathology.

1 Wilson ME, Eusitis HS, Parks MM. Brown's syndrome. Surv Ophthalmol 1989;34:153-72.

2 Goldhammer Y, Lawton Smith J. Acquired intermittent Brown's syndrome. Neurology 1974;24:666-8.

3 Kemp AS, Searle C, Horne S. Transient Brown's syndrome in juvenile chronic arthritis. Ann Rheum Dis 1984;B43:764-5.

4 Brown HW. Congenital structural muscle anomalies. In: Symposium on strabismus, Transactions of the New Orleans Academy of Ophthalmology. St Louis: CV Mosby, 1950: 205-36.

5 Killain PJ, McClain B, Lawless OJ. Brown's syndrome: an unusual manifestation of rheumatoid arthritis. Arth Rheum 1977;20:1080-3. 